\section{Review: bupropion increases abstinence from smoking without affecting mental state in people with schizophrenia}

\section{QUESTION}

Question: Is bupropion safe and effective for achieving abstinence from smoking and reducing nicotine dependence in people with schizophrenia?

Outcomes: Smoking cessation at the end of treatment and at 6 months after the start of treatment: measured by self-report and/or biochemical verification (expired carbon monoxide (CO) level); reduction in nicotine dependence at the end of treatment and at 6 months (measured by change in expired CO level and self-reported number of cigarettes smoked, and other biochemical measurements such as serum cotinine levels); change in positive, negative and depressive symptoms at the end of treatment measured using validated tools; adverse events.

\section{METHODS}

Design: Systematic review with meta-analysis.

Data sources: Cochrane Central Register of Controlled trials, MEDLINE, EMBASE and PsycINFO, from inception to 7 March 2009. Reference lists of relevant studies were also searched to identify unpublished studies, conference abstracts and trial records held by GlaxoSmithKline (manufacturer of bupropion).

Study selection and analysis: Randomised clinical trials (RCTs) comparing bupropion, alone or combined with other pharmacological or non-pharmacological treatments, versus control in adult smokers with schizophrenia (ICD-10 or DSM-IV criteria) were included. Studies involving people with schizophrenia who had nicotine addiction and co-morbid substance abuse disorders were also included. One of the authors searched the databases; two of the authors reviewed the retrieved publications and independently abstracted data from included studies. Authors of the studies were contacted to obtain missing data where necessary. Dichotomous outcomes were expressed as RRs using the Mantel-Haenszel method (random effects). Trial quality was assessed using four criteria: allocation concealment, blinding, completeness of follow-up and whether or not an intention-to-treat analysis was applied. Heterogeneity was investigated using the $\mathrm{I}^{2}$ statistic and the Cochrane $Q$ test.

\section{MAIN RESULTS}

Seven RCTs (21 reports; 260 participants, 10-59 per RCT) were included in the review. Two RCTs compared bupropion plus NRT plus GPT versus placebo plus NRT plus GPT, and four RCTs compared bupropion plus GPT versus placebo plus GPT. One RCT compared bupropion versus placebo but did not mention any co-interventions. Bupropion doses were $150 \mathrm{mg}$ daily or 300 $\mathrm{mg}$ daily, with some trials starting participants on the lower dose and increasing to the higher dose. Duration of treatment ranged from 10 to 12 weeks, and follow-up varied from 7 weeks to 2 years. Overall, bupropion significantly increased biochemically verified self-reported abstinence at the end of treatment and at 6 months after the start of treatment compared with controls (end of treatment: 6 RCTs, 260 people, RR 2.57, 95\% CI 1.35 to 4.88, $p=0.004$; 6 months: 5 RCTs, 214 people, RR 2.78 , 95\% CI 1.02 to $7.58, p=0.05)$. Expired $\mathrm{CO}$ levels were significantly reduced at the end of treatment with buproprion but not at 6 months after treatment (3 RCTs, 123 participants; end of treatment: -6.8 ppm, $95 \%$ CI -11.1 to $-2.6 \mathrm{ppm}, \mathrm{p}=0.002, \mathrm{I}^{2}=0 \%$; at 6 months: $-5.7 \mathrm{ppm}, 95 \% \mathrm{CI}-18.1$ to $\left.+6.6, \mathrm{p}=0.37, \mathrm{I}^{2}=83 \%\right)$. There was no significant difference between bupropion and controls in positive, negative or depressive symptoms (see table 1). Adverse effects: One trial found dry mouth was significantly more common with bupropion than with control $(p<0.05)$. Other adverse effects that showed significantly differences with buproprion in a second study were light-headedness, muscle stiffness, jitteriness and frequent nocturnal wakening.

\section{CONCLUSIONS}

Bupropion reduces nicotine dependence and increases smoking cessation compared with control in people with schizophrenia, without affecting positive, negative or depressive symptoms.

\section{ABSTRACTED FROM}

Tsoi DT, Porwal M, Webster AC. Efficacy and safety of bupropion for smoking cessation and reduction in schizophrenia: systematic review and meta-analysis. Br J Psychiatry 2010;196:346-53.

Correspondence to: Daniel Tai-yin Tsoi, Division of Psychiatry, A Floor, South Block, Queen's Medical Centre, Nottingham NG7 2UH, UK; t.tsoi@sheffield.ac.uk

Sources of funding: Not stated.

Notes, references and a table are published online at http://ebmh.bmj.com
Ith soi and colleagues have definitively shown with the first meta-analysis of smoking cessation treatment trials in schizophrenia what seven small randomised clinical trials showed tentatively: in smokers with schizophrenia, bupropion, with or without nicotine replacement therapy (NRT), doubles to triples the odds of abstinence. This holds both at end of treatment and at 6-month follow-up, while not changing positive, negative or depressive symptoms. Their review is timely. It addresses the neglected smoking epidemic in schizophrenia and reaches the same conclusions as the independently conducted, $\mathrm{NIMH}$ funded, evidence-based 2009 Schizophrenia Patient Outcomes Research Team Psychopharmacological Treatment Recommendations, ${ }^{1}$ which found that people with schizophrenia who want to quit or reduce cigarette smoking should be offered treatment with bupropion SR $150 \mathrm{mg}$ twice daily for 10-12 weeks along with behavioural support, with or without
NRT, to achieve short-term abstinence. With the added power of the meta-analysis, Tsoi and colleagues also find clear evidence for the first time for a benefit of a 12-week course of bupropion for abstinence at 6 months.

While smokers with schizophrenia lose on average 25 years of life to smoking-attributable mortality, in many treatment settings nicotine dependence treatment is not routinely prescribed and in some cases is not reimbursed. In one of the first treatment guidelines for smoking cessation in schizophrenia, Strasser and colleagues called upon physicians to address the public health hazard inherent in the smoking epidemic among those with schizophrenia. They urged smoking cessation within this population to improve quality of life and longevity, but in absence of data, they warned against the use of bupropion. Their theoretical rationale was that use of a dopamine agonist, however weak, may worsen psychotic symptoms. ${ }^{2}$ Thus, we are grateful to Tsoi and colleagues for synthesising the more recent evidence that bupropion confers benefit for smoking cessation with no evidence for harm in smokers with schizophrenia. Psychiatrists and primary care physicians who treat smokers with schizophrenia now have an opportunity to do right by their patients with evidence that these first line pharmacotherapies for nicotine dependence in the general population are both safe and effective for smoking cessation in schizophrenia. We can now issue a call to policy makers to make such treatments routinely available to this population.

\section{A Eden Evins}

Center for Addiction Medicine, Massachusetts General Hospital, Boston, Massachusetts, USA

Competing interests EE has received honoraria from Pfizer and Boehringer Ingelheim. She has also received research grant support from Pfizer and GlaxoSmithKline. 